\title{
Reducing Communication Overhead for Cooperative Localization Using Nonparametric Belief Propagation
}

\author{
Vladimir Savic and Santiago Zazo
}

\section{Linköping University Post Print}

N.B.: When citing this work, cite the original article.

(C)2012 IEEE. Personal use of this material is permitted. However, permission to reprint/republish this material for advertising or promotional purposes or for creating new collective works for resale or redistribution to servers or lists, or to reuse any copyrighted component of this work in other works must be obtained from the IEEE.

Vladimir Savic and Santiago Zazo, Reducing Communication Overhead for Cooperative Localization Using Nonparametric Belief Propagation, 2012, IEEE Wireless Communication Letters, (1), 4, 308-311.

http://dx.doi.org/10.1109/WCL.2012.042512.120172

Postprint available at: Linköping University Electronic Press http://urn.kb.se/resolve?urn=urn:nbn:se:liu:diva-81077 


\title{
Reducing Communication Overhead for Cooperative Localization using Nonparametric Belief Propagation
}

\author{
Vladimir Savic, Student Member, IEEE, and Santiago Zazo
}

\begin{abstract}
A number of methods for cooperative localization has been proposed, but most of them provide only location estimate, without associated uncertainty. On the other hand, nonparametric belief propagation (NBP), which provides approximated posterior distributions of the location estimates, is expensive mostly because of the transmission of the particles. In this paper, we propose a novel approach to reduce communication overhead for cooperative positioning using NBP. It is based on: i) communication of the beliefs (instead of the messages), ii) approximation of the belief with Gaussian mixture of very few components, and iii) censoring. According to our simulations results, these modifications reduce significantly communication overhead while providing the estimates almost as accurate as the transmission of the particles.
\end{abstract}

Index Terms-Cooperative localization, nonparametric belief propagation, communication cost, censoring, message approximation

\section{INTRODUCTION}

Cooperative localization in wireless networks is an important problem, as the availability of location estimate can enable many applications [1]-[3], such as firefighters localization, asset tracking, emergency services and search-and-rescue. We consider the case in which some small number of sensors, called anchor nodes, obtain their coordinates via GPS or by installing them at points with known coordinates, and the rest, target nodes, must determine their own coordinates using the anchor nodes, and measured inter-sensor distances. Distances can be obtained [1], [2], [4] using time of arrival (TOA), time difference of arrival (TDOA), or received signal strength (RSS). If reference sensors were capable of highpower transmission, they would be able to make measurements with all anchor nodes (single-hop positioning). However, it is preferable to use energy-conserving devices without energy necessary for long-range communication. In this case, each sensor only has available noisy measurements of its distance to several neighboring sensors (not necessarily anchor nodes). This technique, also known as multi-hop (or cooperative) localization [1], [3], [5] is fully distributed since each target

Manuscript received March 8, 2012; accepted April 4, 2012.

The authors are with the Signal Processing Applications Group, Universidad Politecnica de Madrid, Madrid, Spain (Email: \{vladimir,santiago\}@gaps.ssr.upm.es).

V. Savic is supported by the FPU fellowship from Spanish Ministry of Science and Innovation; This work is supported, in part, by the Spanish Ministry of Science and Innovation under the grants TEC2009-14219C03-01 and TEC2010-21217-C02-02-CR4HFDVL; program CONSOLIDERINGENIO 2010 under the grant CSD2008-00010 COMONSENS; the European Commission under the grant FP7-ICT-2009-4-248894-WHERE-2. node is responsible to locate itself using only information from its neighborhood.

In the state-of-the-art, there are a lot of methods for cooperative localization (see [1], [6]-[8] and references therein), but most of them provide only location estimate, without associated uncertainty. These methods are known as deterministic (or non-Bayesian) methods. On the other hand, wellknown Bayesian method, nonparametric belief propagation (NBP) [9], provides approximated posterior distributions of the location estimates (as set of weighted particles). However, it is expensive mostly because of the transmission of the particles. In this paper, we propose a novel approach to reduce communication overhead for cooperative positioning based on NBP. It is based on: i) communication of the beliefs (instead of the messages), ii) approximation of the belief with Gaussian mixture of very few components, and iii) censoring (i.e., only informative data have been transmitted). To the best of our knowledge, these ideas are not already applied for this application. According to our simulations results, these modifications reduce significantly communication overhead while providing the estimates almost as accurate as the transmission of the particles (even in the presence of outliers).

\section{COOPERATIVE Localization USING NonParametric BELIEF PROPAGATION}

Let us assume that we have $N_{s}$ sensors ( $N_{a}$ anchors and $N_{t}$ targets) scattered in some region. The 2D location of sensor $r$ is denoted by $x_{r}$. The target node $u$ obtains a noisy measurement $d_{r u}$ of its distance from some neighboring node $r:$

$$
d_{r u}=\left\|x_{r}-x_{u}\right\|+v_{r u} \quad v_{r u} \sim p_{v}
$$

where, for the noise $v_{r u}$, we can assume that $p_{v}$ is Gaussian (for TOA), log-Gaussian (for RSS), or empirical distribution obtained by experimentation in appropriate environment [10], [11]. This measurement provides us likelihood function $p\left(d_{r u} \mid x_{r}, x_{u}\right)$ between each pair of neighboring sensors $(r, u)$. In addition, each sensor $r$ has some prior distribution denoted $p_{r}\left(x_{r}\right)$. Then, the joint posterior probability density distribution (PDF) is given by:

$$
p\left(x_{1}, \ldots, x_{N_{t}} \mid\left\{d_{r u}\right\}\right) \propto \prod_{(r, u) \in E} p\left(d_{r u} \mid x_{r}, x_{u}\right) \prod_{r \in V} p_{r}\left(x_{r}\right)
$$

where $(\mathrm{V}, \mathrm{E})$ is an undirected graph which consists of a set of nodes (or vertices) $V$, and a set of edges $E$. We also need 
to define detection area. For large-scale sensor networks, it is reasonable to assume that only a subset of pairwise distances will be available, primarily between sensors which are located within the some radius $R$. For simplicity, we use this model, but better approximations can be also used [9].

Our goal is to compute the posterior marginal PDF $p\left(x_{n} \mid\left\{d_{r u}\right\}\right)$ (for each target node $n$ ) by marginalizing the joint PDF. However, it is not tractable for the localization problem since the computational time grows exponentially with the number of targets. Therefore, we resort to message passing methods, which are usual choice [9], [12] to approximately solve such problems.

\section{A. Belief propagation (BP)}

Belief propagation [12] is a way of organizing the "global" computation of marginal beliefs in terms of smaller local computations within the graph. It is one of the best-known message passing methods for distributed inference in many applications. The whole computation takes a time proportional to the number of links in the graph, which is significantly less than the exponentially large time that would be required to compute posteriors marginals naively.

For cooperative localization, we choose Markov Random Field (MRW) as underlying graphical model. In that case, we can compute the joint PDF as product of potentials over each of the graph's cliques [9]. For localization, we only need potential functions defined over variables associated with single nodes and pairs of nodes. Single-node potential $\psi_{r}\left(x_{r}\right)$ (prior information about position) at each node $r$, and the pairwise potential (likelihood function $\psi_{r u}\left(x_{r}, x_{u}\right)=p\left(d_{r u} \mid x_{r}, x_{u}\right)$ ) between nodes $r$ and $u$, are respectively given by:

$$
\begin{gathered}
\psi_{r}\left(x_{r}\right)=p_{r}\left(x_{r}\right), \\
\psi_{r u}\left(x_{r}, x_{u}\right)=p_{v}\left(d_{r u}-\left\|x_{r}-x_{u}\right\|\right) .
\end{gathered}
$$

Using (2), we can write the joint posterior PDF, as function of potentials:

$$
p\left(x_{1}, \ldots, x_{N_{t}} \mid\left\{d_{r u}\right\}\right) \propto \prod_{r \in V} \psi_{r}\left(x_{r}\right) \prod_{(r, u) \in E} \psi_{r u}\left(x_{r}, x_{u}\right)
$$

Now we can marginalize this PDF using BP, and use the mean value of this marginal and its associated uncertainty to characterize the target positions. We will use iterative form of BP algorithm [9], in order to adapt it to the localization scenario, where it is more practical to compute beliefs in each iteration, and use them to update messages. The belief (approximation of the posterior marginal $\mathrm{PDF}^{1}$ ) at a node $r$ at iteration $i\left(M_{r}^{i}\left(x_{r}\right)\right)$ is proportional to the product of the local evidence at that node $\psi_{r}\left(x_{r}\right)$, and all the messages coming into node $r$ :

$$
M_{r}^{i}\left(x_{r}\right) \propto \psi_{r}\left(x_{r}\right) \prod_{u \in G_{r}} m_{u t}^{i}\left(x_{r}\right)
$$

where $x_{r}$ is a state of node $r$, and $G_{r}$ denotes the neighbors of node $r$. The messages are determined by the message update

\footnotetext{
${ }^{1}$ In case of tree-like graphs, we can obtain exact marginals.
}

rule:

$$
m_{u r}^{i}\left(x_{r}\right) \propto \int_{x_{u}} \psi_{u r}\left(x_{r}, x_{u}\right) \frac{M_{u}^{i-1}\left(x_{u}\right)}{m_{r u}^{i-1}\left(x_{u}\right)} d x_{u}
$$

In the first iteration of this algorithm it is necessary to initialize $m_{u r}^{1}=1$ and $M_{r}^{1}=p_{r}$ for all $u, r$, and then repeat computation using (6) and (7) until sufficiently converge. It is usually sufficient to run until all unknown nodes obtain information from minimum 3 non-collinear anchor nodes. For the prior of node $r$, we use uniform distribution within the bounded box, in which the node $r$ is localized [10], [13].

Due to the presence of nonlinear relationships and potentially (highly) non-Gaussian uncertainties, we have to use nonparametric version of BP (NBP). Hence, the beliefs and message update equations, (6) and (7), are performed using particle-based approximations (more details in [9], [10]).

In contrast to non-Bayesian approaches, the main advantage of NBP is the ability to provide information about location estimation uncertainties, which are not necessarily Gaussian. Furthermore, it is a naturally distributed method, and it converges after a very small number of iterations. However, BP (and NBP) are not exact in networks with loops [12] due to the double counting problem, i.e., a situation in which the same evidence (as part of the message) is passed around the network multiple times and mistaken for new evidence. This will likely cause overconfident beliefs of the position estimates. Some solutions for this problem can be found in [10], [14], [15] and references therein.

Although mobile positioning is beyond the scope of this paper, NBP can be easily extended. There are two well-known frameworks in the state-of-the-art: one based on factor graph [16], and other based on Markov Random Field [17]. The latter one is sufficient for the distance-based method (as in our case). However, the model based on factor graph is more general, since it can model higher-order interactions (not only pairwise).

\section{REDUCING COMMUNICATION OVERHEAD}

Our goal is to decrease communication cost by: i) communicating beliefs instead of the messages, ii) approximating the packages without significant effect on localization performance, and iii) censoring, i.e., avoiding the transmission of uninformative data.

\section{A. Transmission of the beliefs}

Naturally, one can assume that the messages should be transmitted between each pairs of the neighboring nodes. However, this will make huge communication overhead since each node would need to send one message to each of the neighbors. Obviously, the main problem of this approach is that it does not take the advantage of the broadcast in sensor network (i.e., the transmitted message is needed at just one neighbor, not all). If we recall equation (7), we can see that the messages are computed using i) current belief of the node which transmits the message, ii) pairwise potential which includes information about distance, and iii) outgoing message from the previous iteration. Since the distance measurements can be obtained 
by each node (by sensing, prior to localization) and stored in memory, they should not be transmitted. However, the problem is that each node has only incoming messages, but it can be easily solved by computing the messages twice: once at source node, and once at destination node. Thus, only particles of the beliefs, which are not available at destination node, should be transmitted. The main benefit of this approach is that each node has to broadcast only one package ${ }^{2}$ instead of $n_{d}$ packages in case of message transmission (where $n_{d}$ is node degree). This is paid by slight increase in computation, since the messages must be computed twice. However, the communication is usually much energy-consuming than computation (according to [1], [18], the energy required to transmit one bit, could be used to execute thousands instructions).

\section{B. Package Approximation}

For the described protocol, we would need to transmit $N_{p}$ particles (i.e., $N_{p}$ weights, and $2 N_{p}$ coordinates). However, we can avoid this using following approximations:

- We resample with replacement in order to avoid transmission of the weights. This is already done as part of NBP algorithm (see [9], [10]).

- The bandwidth (which controls the variance of the kernel density estimate of the beliefs [9], [10]) can be computed using unweighted set of particles, so it should not be transmitted.

- We approximate unweighted particles with Gaussian mixture, and transmit only their parameters. Upon receive, we re-draw set of particles from this mixture.

Obviously, first two approximations, practically do not affect accuracy. Regarding last approximation, we expect that (given sufficient mixture components) it will not affect significantly the localization performance. Since the main problem of cooperative localization is the presence of multimodal beliefs (caused by non-rigid graphs and/or multi-modal measurement noise), we expect that Gaussian mixture of very few components is appropriate choice. We cluster unweighted set of particles using standard k-means algorithm [19].

\section{Package censoring}

Additional approximations can be done using package censoring, i.e., avoiding transmission of the packages which provide very small amount of information. To that end, we do the following:

- At first iteration, we only transmit the bounds of the bounded box (i.e., 4 scalar values, which define the rectangle). Then, the particles can be drawn at destination node.

- We do not transmit beliefs in last iterations since they will never be used to update messages.

- Packages from anchor nodes are never transmitted (except their coordinates, if not known in advance).

\footnotetext{
${ }^{2}$ We use term "packages" for the scalar data that will be transmitted, in contrast to term "messages" which refers to NBP messages, which are (in proposed protocol) never transmitted.
}

- We do not transmit beliefs at iteration $i$ which are similar to the beliefs in iteration $i-1$. The similarity can be measured using Kullback Leibler divergence (KLD). If KLD between these beliefs is smaller than predefined threshold $\delta_{K L D}$, the belief should not be transmitted.

- We do not transmit parameters of mixture components which have very small weights (less than predefined threshold $\delta_{w}$ ).

We expect that these steps will reduce significantly the communication overhead. The parameters $\delta_{K L D}$ and $\delta_{w}$ are application dependent, and can be used to make trade-off between accuracy and the cost.

\section{Simulation Results}

We conducted several simulations to analyse the the effect of package approximation and censoring. We assume that there are $N_{a}=4$ anchor nodes and $N_{t}=10$ target nodes, deployed in $100 \mathrm{~m} \times 100 \mathrm{~m}$ area. The anchors are deterministically placed (near the edges of the deployment area). The parameters are set to the following values: number of particles $N_{p}=500$, communication radius $R=20 \mathrm{~m}$, number of iterations $N_{\text {iter }}=5$, thresholds $\delta_{K L D}=0.2, \delta_{w}=0.01$, and number of Monte Carlo runs $N_{M C}=200$. The parameters of the measurement noise (which is two-component Gaussian mixture) are as follow: $\mu_{d_{r u}}=\left(0,0.3 d_{r u}\right), \sigma_{d}=(1 \mathrm{~m}, 1 \mathrm{~m})$ and $w_{d}=(0.6,0.4)$. Thus, the second component of the Gaussian mixture is outlier proportional to the true distance.

We start by analysing the KLD between approximated belief and particle-based belief w.r.t. number of mixture components $N_{m}$. According to Figure 1, we can see that KLD is decreasing as we increase $N_{m}$, as expected. In case of Gaussian noise, we just need 3 or 4 mixture components ${ }^{3}$, but if we add outliers, we will need few more mixture components. To see how this approximation affects the error, we analyse the cumulative distribution function (CDF) for different approximations of the beliefs (particle-based, and beliefs represented with $N_{m}=1$, $N_{m}=3, N_{m}=5$ mixture components). As we can see in Figure 2, 5-mixture approximation achieve almost the same accuracy as particle-based approximation. However, number of mixture components should be a tuning parameter, which will allow the user to make trade-off between accuracy and cost. Note also that package censoring proposed in Section III-C does not affect accuracy at all (i.e., we obtain almost the same curves as in Figure 2).

Finally, assuming $N_{m}=5$, we analyse the communication cost per node. According to Table I, we can conclude the following:

- Transmission of the belief decrease the cost $n_{d}=2.55$ times ( $n_{d}$ is node degree, excluding anchors).

- Mixture approximation significantly decreases communication cost $(96 \%)$. In our case (with $N_{m}=5$ ), we just need to transmit parameteres: mean values $\left(2 N_{m} N_{\text {iter }}=\right.$ 50 packages $)$, the variances $\left(2 N_{m} N_{\text {iter }}=50\right.$ packages, assuming diagonal covariance matrix), and the weights $\left(\left(N_{m}-1\right) N_{\text {iter }}=20\right.$ packages $)$.

\footnotetext{
${ }^{3}$ Note that Gaussian noise does not lead to Gaussian posteriors due to the nonlinearity. In non-rigid graph, they can be multi-modal or circular.
} 


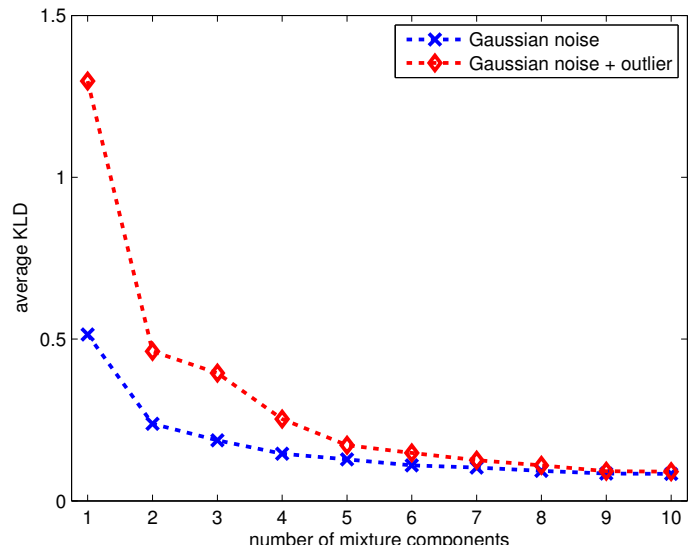

Fig. 1. Kullback-Leibler divergence (KLD) between approximated belief and particle-based belief w.r.t. number of mixture components.

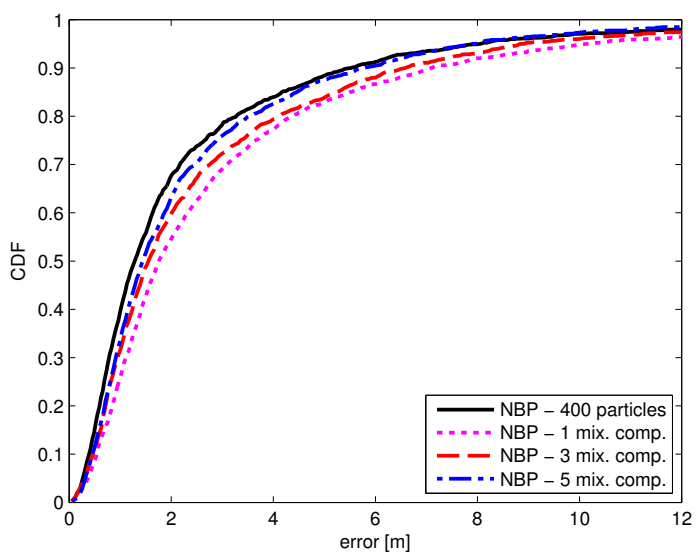

Fig. 2. Cumulative distribution function (CDF) of the position error for different approximations (with or without censoring).

TABLE I

NUMBER OF TRANSMITTED PACKAGES $\left(N_{\text {pack }}\right)$ FOR DIFFERENT APPROXIMATIONS.

\begin{tabular}{c||c} 
Package & $N_{\text {pack }}$ \\
\hline message & 7650 \\
\hline belief (without approx.) & 3000 \\
\hline belief (5-mix approx.) & 120 \\
\hline belief (5-mix approx. and censoring) & 64
\end{tabular}

- Censoring decreases the number of packages nearly to half, in our case. In it expected, especially because of neglectable transmission in the first and last iteration, and also because of the censoring of the beliefs in iteration 4 (in which there are a lot of similar beliefs to previous iteration).

\section{Conclusions}

In this article, we proposed a novel approach to reduce communication overhead for NBP-based cooperative localization. This approach enable the use of NBP in low-cost applications, in which the battery life is critical. Our approach decreases significantly communication overhead while keeping almost the same accuracy of the location estimates. According to our results, it is sufficient to use 3-5 mixture components, even in the presence of outliers. For the future research, it would be interesting to see how proposed approximation works in different applications of NBP. Moreover, hardware implementation could be useful in order to more precisely analyse how communication affects the energy consumption.

\section{REFERENCES}

[1] N. Patwari, J. N. Ash, S. Kyperountas, A. O. Hero, III, R. L. Moses, and N. S. Correal, "Locating the nodes: cooperative localization in wireless sensor networks," IEEE Signal Processing Magazine, vol. 22, pp. 54-69, July 2005

[2] A. Sayed, A. Tarighat, and N. Khajehnouri, "Network-based wireless location: challenges faced in developing techniques for accurate wireless location information," IEEE Signal Processing Magazine, vol. 22, pp. 24-40, July 2005.

[3] M. Z. Win, A. Conti, S. Mazuelas, Y. Shen, W. M. Gifford, D. Dardari, and M. Chiani, "Network localization and navigation via cooperation," IEEE Communications Magazine, vol. 49, pp. 56-62, May 2011.

[4] D. Dardari, A. Conti, U. Ferner, A. Giorgetti, and M. Z. Win, "Ranging with ultrawide bandwidth signals in multipath environments," Proceedings of the IEEE, vol. 97, no. 2, pp. 404-426, 2009.

[5] Y. Shen, H. Wymeersch, and M. Z. Win, "Fundamental limits of wideband localization - part II: Cooperative networks," IEEE Transactions on Information Theory, vol. 56, no. 10, pp. 4981-5000, 2010.

[6] U. A. Khan, S. Kar, and J. M. F. Moura, "DILAND: An algorithm for distributed sensor localization with noisy distance measurements," IEEE Transactions on Signal Processing, vol. 58, no. 3, pp. 1940-1947, 2010.

[7] P. Biswas, T.-C. Liang, K.-C. Toh, Y. Ye, and T.-C. Wang, "Semidefinite programming approaches for sensor network localization with noisy distance measurements," IEEE Transactions on Automation Science and Engineering, vol. 3, no. 4, pp. 360-371, 2006.

[8] S. Srirangarajan, A. Tewfik, and Z.-Q. Luo, "Distributed sensor network localization using SOCP relaxation," IEEE Transactions on Wireless Communications, vol. 7, no. 12, pp. 4886-4895, 2008.

[9] A. T. Ihler, J. W. I. Fisher, R. L. Moses, and A. S. Willsky, "Nonparametric belief propagation for self-localization of sensor networks," IEEE Journal on Selected Areas in Communications, vol. 23, pp. 809819, April 2005.

[10] V. Savic, A. Poblacion, S. Zazo, and M. Garcia, "Indoor positioning using nonparametric belief propagation based on spanning trees,' EURASIP Journal on Wireless Communications and Networking, vol. 2010, pp. 1-12, Aug. 2010.

[11] A. Conti, M. Guerra, D. Dardari, N. Decarli, and M. Z. Win, "Network experimentation for cooperative localization," IEEE Journal on Selected Areas in Communications, vol. 30, no. 2, pp. 467-475, 2012.

[12] J. Pearl, Probabilistic Reasoning in Intelligent Systems: Networks of Plausible Inference. San Mateo: Morgan Kaufmann, 1988.

[13] A. Baggio and K. Langendoen, "Monte carlo localization for mobile wireless sensor networks," Ad Hoc Networks, vol. 6, pp. 718-733, July 2008.

[14] H. Wymeersch, F. Penna, and V. Savic, "Uniformly reweighted belief propagation for estimation and detection in wireless networks," IEEE Transactions on Wireless Communications, pp. 1-9, 2012.

[15] S. Mazuelas, Y. Shen, and M. Z. Win, "Information coupling in cooperative localization," IEEE Communications Letters, vol. 15, no. 7, pp. 737-739, 2011.

[16] H. Wymeersch, J. Lien, and M. Z. Win, "Cooperative localization in wireless networks," Proc. of the IEEE, vol. 97, no. 2, pp. 427-450, 2009.

[17] J. Schiff, E. B. Sudderth, and K. Goldberg, "Nonparametric belief propagation for distributed tracking of robot networks with noisy interdistance measurements," in Proc. of IEEE/RSJ Int. Conf. on Intelligent Robots and Systems, pp. 1369-1376, 2009.

[18] J. C. Chen, K. Yao, and R. E. Hudson, "Source localization and beamforming," IEEE Signal Processing Magazine, vol. 19, pp. 30-39, March 2002.

[19] K. Krishna and M. Narasimha Murty, "Genetic k-means algorithm," IEEE Transactions on Systems, Man, and Cybernetics, Part B: Cybernetics, vol. 29, no. 3, pp. 433-439, 1999. 